Results 1. In addition to expressing characteristic hHCN4 protein, mHCN4-transfected hMSCs also express an anticipated high level of hHCN4 gene by RT-PCR and Western blot analysis. And immunofluorescence image is shown for GFP. Control MSCs were negative. 2. With the use of the whole cell configuration of the patch-clamp technique, $\mathrm{I}_{\mathrm{f}}$ was elicited using hyperpolarizing steps in $10-\mathrm{mV}$ increments from $-40 \mathrm{mV}$ to $-140 \mathrm{mV}$ from a holding potential of $-40 \mathrm{mV}$ and was voltage-dependent. The threshold of voltage for activation of $I_{f}$ is around $-80 \mathrm{mV}$. Remarkably, all hHCN4 positive cells exhibit a large caesium-sensitive $\mathrm{I}_{\mathrm{f}}$ and it was significantly inhibited by $4 \mathrm{mM}$ caesium chloride.

Conclusions The pacemaker current of $\mathrm{I}_{\mathrm{f}}$ can be elicited from the mesenchymal stem cells transfected with $\mathrm{HCN} 4$ genes by LentiV. The genetically engineered MSC expressing hHCN4 is a demonstration of feasibility of preparing MSC-based biological pacemaker cells.

\section{e0032 ROLE OF ATRIAL SUBSTRATE REMODELLING IN INDUCIBILITY OF ATRIAL FIBRILLATION AFTER EPICARDIAL GANGLIONIC PLEXI ABLATION}

doi:10.1136/hrt.2010.208967.32

Zhao Qingyan, Huang He, Zhang Yuguo, Deng Hongping, Yu Shengbo, Huang Congxin. Renmin Hospital of Wuhan University

Objective Investigating the long-term effect of ganglionated plexi (GP) ablation on atrial fibrillation (AF) after GP ablation.

Methods 13 dogs were randomly divided into sham-operated group and GP ablation group. All animals underwent a right thoracotomy at the 4th intercostal space. Induced AF and atrial effective refractory period (AERP) were measured by burst rapid pacing at right atrium. After anterior right GP and inferior right GP ablation, AF and AERP were measured again in the GP ablation group. The animals were allowed to recover for 8 weeks, after which, AF and AERP was measured again. The levels of atrial natriuretic peptide (ANP), TNF-a and interleukin (IL)-6 in blood and atrial tissues were examined. Immunocytochemical staining of cardiac nerves was performed in tissues from the dogs.

Results AF was easily induced in the GP ablation group after 8 weeks while AF was not observed in the sham-operated group, and immediately after GP ablation. AERP and dispersion of AERP (dAERP) were increased after GP ablation, while AERP recovered after 8 weeks. Compared with sham-operated group, the levels of ANP, TNF-a and IL-6 in the right atrium increased significantly 8 weeks after GP ablation $(204.6 \pm 31.2$ vs $299.1 \pm 32.5 ; 1.3 \pm 0.5$ vs $4.7 \pm 0.7 ; 0.9 \pm 0.3$ vs $1.8 \pm 0.5 ; \mathrm{p}<0.05)$. In GP ablation group, the density of GAP43-positive, TH-positive and ChAT-positive nerves in the right atrium was $821 \pm 752,481 \pm 627$ and $629 \pm 644$ per $\mathrm{mm}^{2}$, respectively, which was significantly $(p<0.01)$ lower than the nerve density in sham-operated tissues $(2590 \pm 841,1752 \pm 605$ and $3147 \pm 886$ per $\mathrm{mm}^{2}$, respectively).

Conclusion Atrial substrate remodelling after GP ablation may be the mechanism of induced AF.

\section{e0033 MECHANISMS OF LOSARTAN ATTENUATES VENTRICULAR REMODELLING AND CARDIAC FUNCTION IN AORTIC BANDED RATS WITH CHRONIC HEART FAILURE}

doi:10.1136/hrt.2010.208967.33

${ }^{1}$ Fu Deming, ${ }^{1}$ Xiao Chuanshi, ${ }^{2}$ Zhu Qiujuan, ${ }^{1}$ Chai Yingru. 'Department of Cardiology The Second Hospital of Shanxi Medical University; ${ }^{2}$ Department of Hematology The Second Hospital of Shanxi Medical University

Objective To investigate the regulation of Losartan (Los) on ventricular remodelling and cardiac functioning chronic heart failure rats induced by aortic banded.
Methods Sprague-Dawley (SD) rats underwent abdominal aorta coarctation to induce CHF, confirmed by ultrasound cardiograph and Catheterisation, or sham operation, followed by 8 weeks treatment with Losartan $(20 \mathrm{mg} / \mathrm{kg}$ per day, orally) or vehicle (drinking water). Plasma norepinephrine (NE) was measured by ELISA, and plasma and tissue angiotensin II (Ang II) levels were measured using RIA. Cardiomyocyte apoptosis was examined by agarose gel electrophoresis and TUNEL's method. The mRNA levels of Bax and $\mathrm{Bcl}-2$ were determined by RT-PCR and the protein expression of phosphorylated and total Akt(t-Akt and p-Akt) were assessed by Western blot.

Results Losartan-treated CHF rats had lower left ventricular enddiastolic pressure (LVEDP) [COA+Los: $(11.47 \pm 5.06) \mathrm{mm} \mathrm{Hg}$ vs COA+Vehicle: $(21.18 \pm 6.56) \mathrm{mm} \mathrm{Hg}, \mathrm{p}<0.01]$, higher left ventricular ejection fraction (LVEF) [COA+Los: $(63.28 \pm 4.32) \%$ vs COA +Vehicle: $(43.27 \pm 4.25) \%, \quad p<0.05]$ and lower left ventricle/body weight ratios $[\mathrm{COA}+$ Los: $(2.41 \pm 0.15) \mathrm{mg} / \mathrm{g}$ vs COA+ Vehicle: $(3.12 \pm 0.18) \mathrm{mg} / \mathrm{g}, \quad \mathrm{p}<0.05]$, lower plasma NE[COA+Los: $(682.24 \pm 64.66) \mathrm{pg} / \mathrm{ml}$ vs COA+Vehicle : (908.24 \pm 75.10$) \mathrm{pg} / \mathrm{ml}$, $\mathrm{p}<0.05]$ and myocardium Ang II[COA+Los : $(60.15 \pm 6.22) \mathrm{pg} /$ mgprot vs COA + Vehicle : $(92.31 \pm 8.31) \mathrm{pg} / \mathrm{mgprot}, \mathrm{p}<0.05]$, but higher plasma Ang II[COA+Los : $(629.68 \pm 85.71) \mathrm{pg} / \mathrm{ml}$ vs COA+ Vehicle : $(475.67 \pm 51.12) \mathrm{pg} / \mathrm{ml}, \mathrm{p}<0.05]$ than vehicle-treated CHF rats. Losartan-treated HF rats had no obviously "DNA ladder" which was the character of apoptosis, and the apoptosis index was also reduced [COA+Los: $(5.49 \pm 0.45) \%$ vs COA+Vehicle: $(9.62 \pm 0.51) \%, \mathrm{p}<0.05]$ with a lower express of $\mathrm{Bax} / \mathrm{Bcl}-2$ gene [COA+Los: $(109.58 \pm 7.53) \%$ vs COA+Vehicle: $(136.76 \pm 8.82) \%$, $\mathrm{p}<0.05]$ and a higher protein expression of $\mathrm{p}$-Akt [COA+Los: $(70.80 \pm 6.40) \%$ vs COA+Vehicle: $(50.30 \pm 4.80) \%, p<0.05]$

Conclusion Losartan might inhibit cardiomyocyte apoptosis and improve cardiac function in aortic banded rats by blocking Ang II to bind AT1-R and promoting the activation of Akt.

Conclusion Los attenuated ventricular remodelling and improve cardiac function in aortic banded rats by blocking angiotensin II to bind $\mathrm{AT}_{1}-\mathrm{R}$ and reducing angiotensin II, aldosterone and inflammatory cytokine in myocardium.

\section{e0034 INHIBITION OF ATORVASTATIN ON THE AUTOPHAGY OF VASCULAR ENDOTHELIAL CELLS}

doi:10.1136/hrt.2010.208967.34

Zhang Sheng-Xue, Qiu Ling, Xiao Chuan-Shi, Fang Ya-Qin. The Second Hospital of Shanxi Medical University

Objective To explore the mechanism of atorvastatin's protection on vascular endothelial cells, we conducted the research of impact of atorvastatin on vascular endothelial cells autophagy in different times.

Methods We used the Hank's to replace the normal medium to induce autophagy of vascular endothelial cells. In pre-inducement and induction procedure, the cells were incubated with normal medium which includes atorvastatin or not and Hank's, respectively. Experimental cells were randomly divided into four groups: control group (Group I), the group of pre-inducement with atorvastatin (Group II), the group of inducement with atorvastatin (Group III) and the group of pre-inducement and inducement both with atorvastatin (Group IV). Transmission electron microscope (TEM) was used to detect autophagy. The RT-PCR was employed to detect the autophagy-specific markers' (Beclin 1 and Map1lc3) expression in each group of cells.

Results Compared with the group I, target genes (Beclin 1 and Map1lc3) of the group III and the group IV had a significant decreased expression $(p<0.01)$. The expression of the group II were all lower than the group I $(p>0.05)$. The group IV was better than the group III $(p>0.05)$. Via TEM detection, the cells showed typical 
morphological change. There into, the proportions of preautophagosome to the total area of cytoplasm in group III and group IV are remarkably decreased than that of group I, and the difference was significant $(p<0.01)$. The proportions of group II is lower than that of group I, but not significant ( $p>0.05$ ). The proportions of group IV is lower than that of group III, but there was no obvious difference between two groups ( $p>0.05)$.

Conclusion Using atorvastatin, in induction procedure, can inhibit vascular endothelial cells autophagy, which may be related to the role of atorvastatin's improvement on endothelial function. However, using atorvastatin, prior to the occurrence of induced autophagy, can not quite inhibit the occurrence of autophagy.

\section{E0035 THE STUDY OF DERIVATION FROM BONE MARROW MESENCHYMAL STEM CELLS INTO CARDIOMYOCYTE-LIKE CELLS IN VITRO VIA CARDIOTROPHIN-1}

doi:10.1136/hrt.2010.208967.35

Chaoquan Peng, Ya Gao, Peng Xiang, Ke Yang, Liyuan Zou, Xiao Wu. The Third Afiliated Hospital of Sun Yat-sen University

Objective To investigate the effects of CT-1 on differentiation of induced swine BMSCs in vitro independently or with 5-aza.

Methods BMSCs were divided into four groups : bland contro1; induced with CT-1; induced with 5-aza; induced with 5-aza combined with CT-1. After 4 weeks of induced culturing, the differentiation of cardiomyocyte induced were estimated by cTnT and a-actinin.

Result Red fluorescence staining of a-actin shows: the differentiation rate of myocardial cells of the composite group is greatest (29.900 $4.7571 \%)$; 5 -aza group is greater than CT-1 group; CT-1 group is greater than control. Red fluorescence staining of cTnT shows: the differentiation rate of myocardial cells of the composite group is greatest $(36.500 \pm 4.0927 \%) ; 5$-aza group is greater than CT-1 group; CT-1 group is greater than control.

Conclusions CT- 1 can increasing the inducing rate commbined witn 5-aza.

\section{e0036 REGULATION OF T-TYPE CA ION CHANNEL IN LYSOPHOSPHATIDYLCHOLINE-STIMULATED CARDIOMYOCYTES}

doi:10.1136/hrt.2010.208967.36

Gang Liu, Li Tian, Zhen-Guo Ji, Chao Liu, Kun-Shen Liu, Ming-Qi Zheng. First Hospital of Hebei Medical University

Objectives To study the effect of lysophosphatidylcholine (LPC) in myocardial cells in T-type calcium channel currents $\left(\mathrm{I}_{\mathrm{CaT}}\right)$, and the hypothesis that LPC accumulation in intracellular and/or interstitial space in cardiomyocytes may underlie as a mechanism for tachycardia and various arrhythmias during cardiac ischaemia.

Methods Neonatal rat cardiomyocytes from 1 to 3-day-old Wistar rats and hypertrophied ventricular myocytes from Wistar rat were prepared. A single dose of $60 \mathrm{mg} / \mathrm{Kg}$ monocrotaline was injected into the intraperitoneal cavity at the age of 8 weeks old, and right ventricular myocytes were isolated enzymatically at the age of 14 weeks from male Wister rats. In this study, whole cell patch clamp cardiac myocyte and heterologous expression of human CaV3.1 and CaV3.2 ion channel components were measured. The effect of LPC through activation of PKC isoforms mediated $\mathrm{I}_{\mathrm{CaT}}$ control mechanisms was studied.

Results (1) LPC markedly accelerated the spontaneous beating rates of neonatal rat cardiomyocytes from $42 \pm 8 \mathrm{bpm}$ in control to $64 \pm 8 \mathrm{bpm}$ after LPC application in $5 \mathrm{~min}$ at the physiological $\left[\mathrm{Ca}^{2+}\right]_{\mathrm{i}}$ condition $(\mathrm{pCa}=7.2)$. (2) In neonatal cardiomyocytes, $\mathrm{I}_{\mathrm{Ca} . \mathrm{T}}$ was significantly increased by $10 \mu \mathrm{M}$ LPC by $21.5 \%$ when $\left[\mathrm{Ca}^{2+}\right]_{\mathrm{i}}$ was high $(\mathrm{pCa}=7)$. Intracellular $\mathrm{Ca}^{2+}$-dependent augmentation of $\mathrm{I}_{\text {Ca.T }}$ by LPC was confirmed not only in neonatal cardiomyocytes but in adult ventricular myocytes from the hypertrophied heart. In this experiment, $\mathrm{I}_{\text {Ca.T }}$ was significantly increased by $10 \mu \mathrm{M}$ LPC by $23.5 \%$ when $\left[\mathrm{Ca}^{2+}\right]_{i}$ was high $(\mathrm{pCa}=7)$, although it was unchanged when $\left[\mathrm{Ca}^{2+}\right]_{\mathrm{i}}$ was low $(\mathrm{pCa}=11)$. (3) LPC exerted no effect on the Cav3.1 T-type $\mathrm{Ca}^{2+}$ channel current $\left(\mathrm{I}_{\text {Cav3.1 }}\right)$ regardless of the $\left[\mathrm{Ca}^{2+}\right]_{\mathrm{i}}$ condition at a $\mathrm{pCa}$ of 7 (solution $\mathrm{F}$ ) or at a $\mathrm{pCa}$ of 11 (solution A). In contrast, LPC upregulated the Cav3.2 T-type $\mathrm{Ca}^{2+}$ channel current ( $\mathrm{I}_{\mathrm{Cav3.2}}$ ), which was much larger at a $\mathrm{pCa}$ of 7 than that at a pCa of 11. (4) A specific PKC $\alpha$ inhibitor Ro-32-0432 completely blocked the effect of LPC on $\mathrm{I}_{\mathrm{Cav} 3.2}$. However, in the same culture condition, a specific PKC $\beta I$ inhibitor Gö 6976 (20 nM) and a specific PKC $B \mathrm{II}$ inhibitor CGP-53353 $(2 \mu \mathrm{M})$ did not modify the effect of LPC on $\mathrm{I}_{\text {Cav3.2. }}$.

Conclusion The present study indicates that intracellular signal PKC $\alpha$ activation by LPC upregulates the cardiac $\mathrm{I}_{\mathrm{Ca} . \mathrm{T}}$ in physiological or higher $\left[\mathrm{Ca}^{2}{ }^{+}\right]_{\mathrm{i}}$ condition may be a novel ischaemia-related mechanism, which may accelerate the pathophysiological cardiac automaticity and trigger tachyarrhythmias.

\section{e0037 EFFECTS OF ENALAPRIL AND IRBESARTAN ON AORTA REMODELLING AND ION PUMPS IN RENOVASCULAR HYPERTENSIVE RATS}

doi:10.1136/hrt.2010.208967.37

${ }^{1}$ Qian-Hui Shang, ${ }^{1}$ Wei Hu, ${ }^{1}$ Qian-Feng Jiang, ${ }^{2}$ Oin Wu, ${ }^{1}$ Ping Yuan. ${ }^{1}$ Institute of Clinical Medicine of Zunyi Medical College, Department of Cardiology, Affiliated Hospital of Zunyi Medical College; ${ }^{2}$ Department of Pharmacology, Zunyi Medical College, Zunyi Guizhou, China

Objective To investigate the effects of single-drug or combination therapy of enalapril and irbesartan on aorta remodelling and its mechanisms.

Methods Renovascular hypertensive rats (RHD) induced by twokidney one-clip method were treated with normal saline (mode group, $\mathrm{n}=6$ ), enalapril [10 $\mathrm{mg} /(\mathrm{kg} \mathrm{d}), \mathrm{n}=6$ ], irbesartan [50 mg/ $(\mathrm{kg} \cdot \mathrm{d}), \mathrm{n}=6$ ] and enalapril+irbesartan [5 mg/ (kg d) $+25 \mathrm{mg} /(\mathrm{kg} \mathrm{d})$ $\mathrm{n}=6]$ for 6 weeks. Six sham-operated rats were used as controls. Aortic morphology and structural changes in the media were observed by $\mathrm{HE}$ staining, immunohistochemistry and Masson staining. The content of Angiotensin II (Ang II) was measured by radioimmunoassay. The activities and mRNA levels of $\mathrm{Na}^{+}$pump and $\mathrm{Ca}^{2+}$ pump in aortic media were determined by enzyme assay and real-time PCR respectively.

Results The media area of aorta and the Ang II content were significantly increased in model group, while the activities and the mRNA levels of $\mathrm{Na}^{+}$pump and $\mathrm{Ca}^{2+}$ pump in aortic media were obviously decreased, and $\mathrm{Na}^{+}$pump and $\mathrm{Ca}^{2+}$ activities were increased in enalapril group and irbesartan group $(p<0.01)$. The Ang II content was obviously decreased in enalapril group, while increased in irbesartan group $(p<0.01)$. The mRNA levels of sodium pump $a_{1}$-subunit and plasma membrane calcium pump isoform 1 (PMCA1) in aorta smooth muscle tissue were significantly increased in enalapril group $(p<0.01)$. The amelioration of blood pressure, $\mathrm{Na}^{+}$ pump and $\mathrm{Ca}^{2+}$ pump activities, media area and thickness in combination group was significantly better than single-drug intervened group $(p<0.01)$.

Conclusion The amelioration of aorta remodelling induced by enalapril and irbesartan may be associated with the increase of $\mathrm{Na}^{+}$ pump and $\mathrm{Ca}^{2+}$ pump activities. There may be some synergistic effects on ameliorating of $\mathrm{Na}^{+}$pump and $\mathrm{Ca}^{2+}$ pump activities and aorta remodelling from combination of the two drugs. The effect of enalapril on $\mathrm{Na}^{+}$pump and $\mathrm{Ca}^{2+}$ pump activities may be mediated by increasing their mRNA expression. 